\title{
Um método para o cálculo do benefício econômico e definição da estratégia em trabalhos de redução do tempo de setup
}

\section{A method for the calculation of economic benefits and development of setup time reduction strategies}

\author{
Mário Eduardo Pauka Reis ${ }^{1}$ \\ João Murta Alves ${ }^{1}$
}

\begin{abstract}
Resumo: Uma das bases da produção enxuta é o baixo tempo de setup, que pode ser obtido por soluções organizacionais de baixo custo e/ou de projeto de alto custo, tal como mudança de máquina. Quando a mudança de projeto se apresentar como a melhor solução, torna-se necessário o cálculo do ganho para se ter certeza da viabilidade econômica da ação. O presente artigo apresenta um método para se calcular o ganho da redução de tempo de setup e mostra em quais situações as soluções de projeto devem ser consideradas como a primeira opção. Um estudo de caso da implementação do método procura evidenciar a sua efetividade.
\end{abstract}

Palavras-chave: Setup. SMED. Lean.

\begin{abstract}
One of the pillars of lean manufacturing is the low setup time that may be obtained by low cost organizational solutions and/or design such as relatively high cost machine modifications. When design change is the best solution, it is necessary to calculate the gains in order to be sure of the economic feasibility of the action. The present article introduces a method to calculate the gains originated from the reduction of setup time, and it defines the situations in which the project solutions may be considered as the first option. A case study was used to investigate the effectiveness of such method.
\end{abstract}

Keywords: Setup. SMED. Lean.

\section{Introdução}

São diferentes as motivações para se reduzir o tempo de setup. Na literatura, observam-se trabalhos para redução de tempo de setup realizados para resolver problemas de capacidade (VOLLMANN et al., 1988) e para implementar a produção enxuta (SEPHERI, 1987).

A redução no tempo de setup para resolver problemas de capacidade tem um ganho fácil de ser calculado, o que geralmente é o gasto evitado para comprar uma nova máquina, ou para implementar um novo turno, mas no caso da implementação da produção enxuta, este ganho não é tão facilmente medido. Por exemplo, Nishida (2006) comenta que reduzir o tempo de setup possibilita trabalhar em pequenos lotes, diminuir os estoques, aumentar a flexibilidade e atender mais rapidamente à demanda dos clientes, mas quando explica como escolher uma meta para redução de tempo de setup, coloca como único ganho calculável a redução do inventário.

Vale salientar que o cálculo do ganho pode não ser necessário se forem obtidas grandes reduções com baixos investimentos, o que é propagado no método SMED (Single Minute Exchange of Die) criado por Shingo (2000), que enfoca medidas organizacionais. Quando soluções organizacionais não são suficientes para reduzir o tempo de setup, pode-se considerar mudanças de projeto que são soluções mais caras, defendidas por McIntosh et al. (2001).

$O$ presente trabalho tem como objetivo ser um guia para evitar que se tomem medidas de redução do tempo de setup organizacionais (caminho mais comum e mais largamente aplicado), quando o mais adequado poderiam ser medidas de "projeto" (mudança de máquina). Isto proporciona a tomada de decisão correta, evitando o desgaste do líder do projeto e aumentando o ânimo da equipe.

Este trabalho está estruturado em 6 Seções. $\mathrm{Na}$ Seção 2, comenta-se o método SMED (Single Minute Exchage of Die), que tem um enfoque organizacional para redução do tempo de setup. Na seção 3, discorre-se sobre trabalhos que defendem a redução do tempo de setup por mudança de equipamentos (projeto). $\mathrm{Na}$

\footnotetext{
${ }^{1}$ Departamento de Organização, Instituto Tecnológico de Aeronáutica, Divisão de Engenharia, Mecânica Aeronáutica, Praça Mal. Eduardo Gomes, 50, CEP 12228-900, São José dos Campos - SP, Brasil, E-mails: mpauka@uol.com.br; murta@ita.br
} 
Seção 4, apresenta-se um método para cálculo do ganho em trabalhos de redução do tempo de setup, que também mostra a expectativa da abordagem para a solução do problema. Na Seção 5, apresenta-se um estudo de caso em que se aplica o método proposto e, na Seção 6, apresentam-se as discussões e conclusões.

\section{O método Single Minute Exchage of Die de redução de tempo de setup}

Shingo (2000) criou um método para diminuir o tempo de setup que, segundo ele, foi desenvolvido em um período de 19 anos como resultado de exames detalhados de aspectos teóricos e práticos. Esse método é denominado SMED.

A base do seu método está no entendimento de que as operações de setup são de dois tipos diferentes:

- setup interno - atividades que só podem ser realizadas quando a máquina estiver parada, por exemplo: montagem ou remoção de matrizes; e

- setup externo - atividades que podem ser realizadas quando a máquina estiver funcionando, por exemplo: transporte de matrizes já utilizadas para o almoxarifado.

O seu método constitui-se de 4 estágios, vistos a seguir, que também são apresentados na Figura 1.

- Estágio 0-As condições de setup interno e externo são confundidas; o que poderia ser realizado externamente é realizado internamente e, por isto, as máquinas ficam paradas por longos períodos.

- Estágio 1 - Separar os setups interno e externo. Já nessa fase, atinge-se uma economia de 30\% a $50 \%$ do tempo de setup.

- Estágio 2 - Converter o setup interno em externo. Exemplo: realizar pré-aquecimento de elementos que anteriormente eram aquecidos após o início do setup e a conversão da centragem em um elemento externo, realizando-a antes do início da produção.

- Estágio 3 - Racionalizar todos os aspectos da operação de setup.

\section{Redução de tempo de setup por foco em projeto}

Outro enfoque, em alguns aspectos bem diferentes de Shingo, foi apresentado por McIntosh et al. (1996), que comentam que os métodos para redução de tempo de setup podem ser separados em baseados em organização e baseados em projeto, e definem "projeto" como atividade, na qual o equipamento é modificado para melhorar o tempo de setup. Comentam também que o método de Shingo é baseado na separação dos setups interno e externo, o que é uma mudança organizacional, e mostram que, apesar de Shingo dar enfoque ao aspecto organizacional, vê-se no seu livro um grande uso da melhoria de tempos de setup por "projeto". Observam ainda que, nos exemplos do livro de Shingo, apenas 36\% das melhorias citadas por ele foram organizacionais.

Eles comentam que poderia ser argumentado que "projeto" é um componente do estágio 3 de Shingo (racionalização das fases do setup), mas seria mais útil considerar o "projeto" separadamente, pois "projeto" tem o efeito de mudar o sistema de manufatura que previamente existia e, consequentemente, impor uma mudança nos procedimentos existentes de troca.

Colocam, então, a seguinte questão: se o "projeto" realmente influencia o desempenho da troca, por que soluções com mudança de máquina não foram

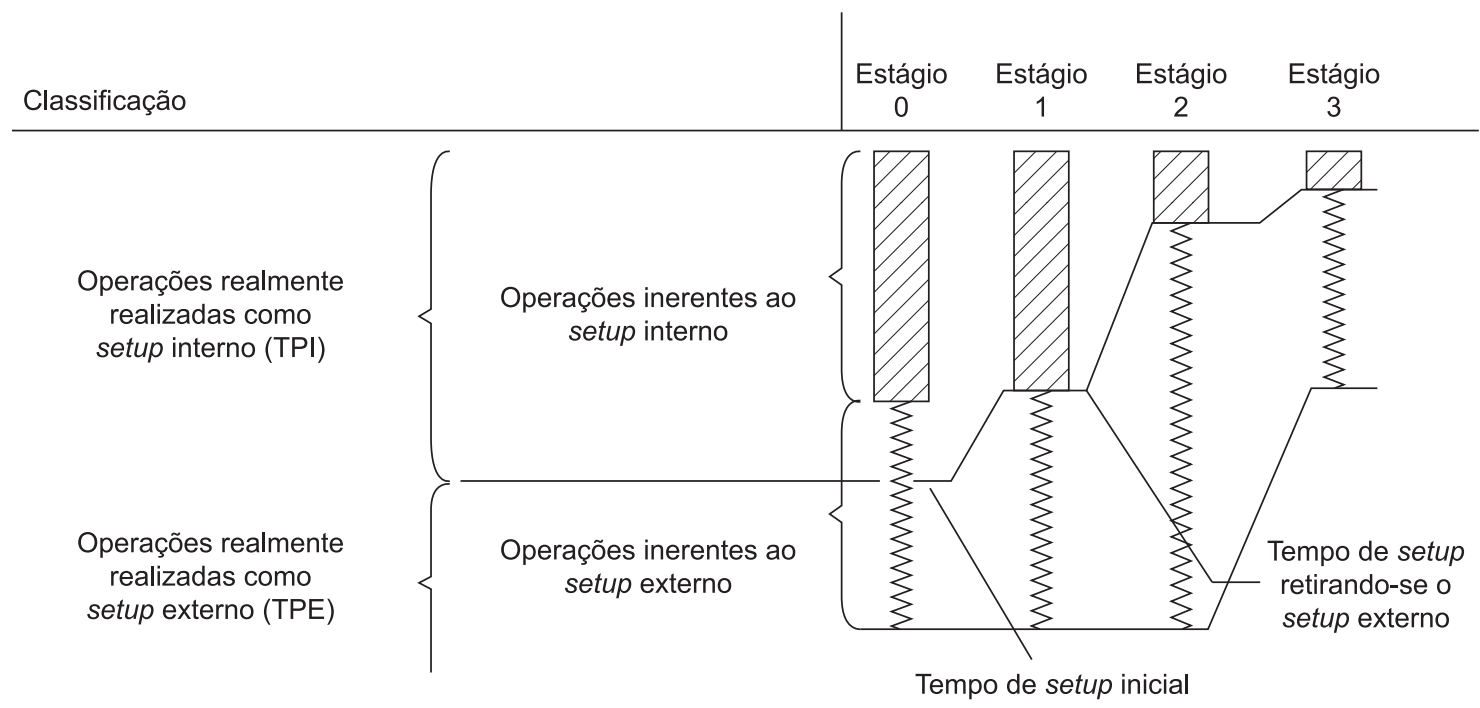

Figura 1. Evolução do setup seguindo-se os estágios do método SMED. Fonte: Shingo (2000). 
promovidas tão vigorosamente? E respondem à própria pergunta.

A resposta talvez esteja no fato de que muitos daqueles que publicaram sobre o setup são consultores (Hay-Rath \& Strong, Mather - Hal Mather Inc., Oishi - JIT Automation Inc., Claunch - ProTech, Johansen and McGuire - Manufacturing Excellence Action Coalition, Steudel and Desruelle - H.J. Steudel $\&$ Associates Inc.). Outros reportaram resultados de melhorias em tempo de setup durante o trabalho com consultores ou logo após (Jones- Productivity Europe, Lee - Rath \& Strong). E é simplesmente natural que firmas de consultoria enfatizem as abordagens que mais se aproximam do conhecimento de seus especialistas. Desta forma, há uma tendência para aspectos organizacionais em detrimento de aspectos de projeto. (McINTOSH et al., 1996).

McIntosh et al. (1996) mostram, no Quadro 1, as diferenças entre as abordagens de diminuir o tempo de setup por mudança organizacional ou por projeto (design).

Segundo McIntosh et al., 1996, há um consenso na indústria de que as dificuldades de setup devem ser tratadas por times de melhoria do local de trabalho, e que essa abordagem tem muitos méritos. Entretanto, os autores afirmam que é totalmente equivocado desqualificar a opção de uma solução baseada em uma substancial mudança de "projeto". Inclusive, neste caso, McIntosh et al. (2001) comentam que as melhorias poderiam ser realizadas por um grupo de profissionais que não são do chão de fábrica como, por exemplo, projetistas de máquinas.

McIntosh et al. (2000) reforçam a crítica ao SMED de focar muito na questão organizacional. Comentam que, antes do estágio final do modelo de Shingo, que é intitulado "racionalizar todos os aspectos da operação de setup" (estágio 3 da Figura 2), ainda não houve alteração da duração das atividades pertinentes ao setup e que a redução do tempo das atividades por "projeto" pode gerar diminuição do tempo de setup pelo menos comparáveis à conversão do setup interno em externo. Exemplos da importância de se focar em "projeto" seriam dados pelo próprio Shingo, quando ele cita que ajustes representam 50\% do tempo de setup.

Mileham et al. (1999), defendendo o enfoque da diminuição de tempo de setup por "projeto", comentam que há três estratégias que podem ser adotadas para reduzir o tempo de setup, que são: "método", "projeto e método" e "projeto".
Estas três estratégias são explicadas a seguir:

- Melhoria por "método": reduz o tempo reorganizando os métodos existentes e fazendo-os mais eficazes;

- "Projeto e método": referem-se a casos em que o tempo se reduziu ainda mais pelo uso de, por exemplo, parafusos de aperto com $1 / 4$ de volta ou estruturas especiais para levantamento de prensas; e

- Melhoria por "projeto": um exemplo seria a instalação de novas prensas automotivas que incorporam princípios para se realizar um setup rápido.

A Figura 2 traz o custo e o impacto no tempo de setup das abordagens: "método", "projeto e método" e "projeto".

Na Figura 2, observa-se que as abordagens "método" e "projeto e método", embora tenham menores custos, impõem limites à redução do tempo de setup.

Uma explicação sobre os custos relacionados à abordagem "método" é apresentada por Gallego e Moon (1995) que explicam que externalizar operações do setup interno resulta em maiores custos de setup porque tais operações, quando feitas fora da máquina, tipicamente consomem mais tempo, requerem operadores adicionais e melhores, ou coordenação mais cuidadosa da gerência.

Vale enfatizar que, apesar de haver um custo no ato de externalizar atividades do setup, esta opção é bastante adotada na Toyota, inclusive tornando o setup externo maior que o setup interno. Monden (1998) esclarece que, embora o setup interno na Toyota tenha diminuído para valores menores que dez minutos, o setup externo ainda requer de 30 minutos a uma hora. Sem a espera deste tempo, não se pode passar para o próximo lote. Como resultado, o tamanho do lote, ou número de setups por dia na Toyota, deve ser determinado pela restrição do tempo gasto no tempo de setup externo.

Gallego e Moon (1995) destacam que esta opção de externalizar setups pode diminuir o tamanho dos lotes com o aumento dos custos do setup, e que esta observação parece estranha quando analisada em relação à teoria do lote econômico, na qual os lotes são proporcionais à raiz quadrada dos custos de setup.

Quadro 1. Diferenças entre as abordagens para diminuir o tempo de setup. Fonte: McIntosh et al. (1996).

\begin{tabular}{|lcc|}
\hline & $\begin{array}{c}\text { Mudança Organizacional } \\
\text { (melhorar práticas existentes) }\end{array}$ & $\begin{array}{c}\text { Mudança por Projeto } \\
\text { (incitar novas práticas) }\end{array}$ \\
\hline Tempo para alcançar a melhoria & Usualmente alcançado rapidamente & Frequentemente uma evolução demorada \\
Esforço requerido & Usualmente de baixo para médio & Usualmente de médio para alto \\
Custo & Usualmente de baixo para médio & Usualmente de médio para alto \\
Manter os ganhos & Requerida uma constante atenção & Relativamente fácil de manter \\
Economia potencial de tempo & $70 \%$ & $100 \%$ \\
\hline
\end{tabular}




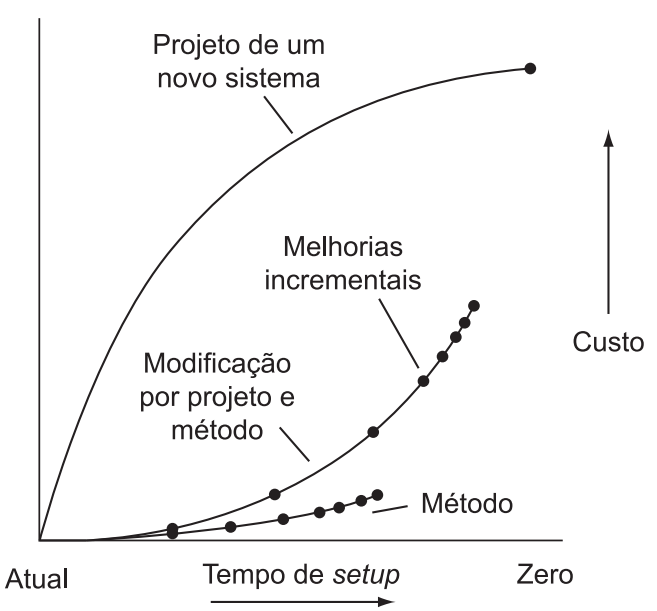

Figura 2. Limites hipotéticos e respectivos custos das estratégias de melhoria de tempo de setup. Fonte: Mileham et al. (1999).

\section{Método para cálculo do ganho na redução de tempo de setup e expectativa de abordagem para solucionar o problema}

Para saber o que se pode incorporar em um ganho advindo de uma redução de tempo de setup, utilizou-se como base uma lista feita por McIntosh et al. (2001) que coloca como ganhos da redução do tempo de setup as cinco áreas a seguir:

- Tempo de parada de equipamentos;

- Inventário;

- Recursos (exemplo: menos necessidade de mão de obra, menos necessidade da habilidade da mão de obra);

- Flexibilidade; e

- Controle do processo (exemplo: aumentar a qualidade do produto, aumentar a confiabilidade do processo).

Por meio dessa lista, será desenvolvido um método de cálculo de ganho que possa incorporar o máximo possível de ganhos calculáveis, advindos do setup. Vale salientar que seria muito importante conseguir todos os ganhos advindos da redução do tempo de setup, pois, se for possível visualizar um maior ganho, haverá mais apoio para se trabalhar no problema e poderá ser considerada a implementação de um leque maior de soluções.

A seguir, será mostrado como calcular o ganho das cinco áreas anteriormente comentadas.

\subsection{Tempo de parada de equipamentos}

Segundo McIntosh et al. (2001), um exemplo do ganho nessa área é o aumento de capacidade para uma demanda de mercado existente. Nesse caso, o ganho é a somatória dos gastos que se evita fazer para aumentar a capacidade, devido à diminuição de tempo de setup. Um exemplo desse caso é observado em um comentário de Clegg (1986), ao citar que a redução em $34 \%$ no tempo de setup evitou a compra de um novo laminador, cujo custo era de US\$ 500.000.

\subsection{Inventário}

Nishida (2006) mostra um exemplo em que a diminuição do tempo de setup para a metade do valor inicial duplica o giro de estoque, reduzindo o espaço físico para a metade.

O cálculo da diminuição do estoque em função da redução do tempo de setup é algo possível de ser realizado e é apresentado por Nishida (2006), Smalley (2004) e Reis (2006).

A transformação da redução de inventário em ganho pode ser feita considerando-se que custo do inventário é dado pelo seu custo de capital. Vale salientar que essa mesma abordagem foi seguida por Fabry e Gelders (1991) que, em um trabalho de melhoria em uma indústria, comentam que uma redução do estoque em DM8,9 milhões/ano, considerando uma taxa de retorno de $21 \%$, gerou uma economia de aproximadamente DM1,85 milhões/ano.

\subsection{Recursos}

Como comentado por McIntosh et al. (2000), reduzir recursos pode vir da economia de mão de obra, disponibilizando técnicos especializados ou possibilitando a contratação de operadores de menor especialização. Outro ganho viria da possibilidade de se utilizar menos equipamentos, não sendo necessário gastar na atualização dos equipamentos em desuso, ou pela criação de espaço em função da retirada desses equipamentos.

Neste trabalho, quando for considerada a redução de recursos, estar-se-á na realidade considerando a redução de mão de obra no local onde é aplicada a melhoria. Decidiu-se por esse enfoque por observar-se que é possível pensar em trabalhar em setup com o objetivo de reduzir mão de obra, como comentado por Hay (1987), mas não se encontrou nenhum trabalho em que o objetivo de redução de tempo de setup fosse diminuir o número de máquinas ou fosse a liberação de área. Esses ganhos, na realidade, parecem ser efeitos colaterais positivos que devem ser contabilizados quando for possível.

Para a área "recursos", o ganho aqui considerado será o gasto não mais realizado pela transferência de mão de obra. É importante enfatizar que demitir mão de obra como consequência da redução do tempo de setup não é apoiado por Hay (1987). Segundo ele, as pessoas que fazem o setup são vitais para o sucesso do trabalho e as melhorias não aconteceriam se essas pessoas acreditassem que suas ideias eliminariam os seus empregos. 
Womack e Jones (2004) concordam com Hay (1987) e comentam que demitir funcionários em uma fábrica com excesso de mão de obra, devido à introdução da produção enxuta, pode criar uma resistência a novas melhorias e sugerem 5 ações para não realizar a demissão:

- reduzir horas extras;

- alocar pessoal excedente nas atividades de Kaizen;

- fabricar internamente alguns componentes produzidos por fornecedores;

- reduzir a semana de trabalho; e

- e a mais poderosa de todas: desenvolver novas linhas de produtos para expandir a empresa.

Em resumo, a melhor forma de reduzir a mão de obra em trabalhos de redução de tempo de setup seria por meio da transferência deste recurso para setores que dele necessitam.

\subsection{Flexibilidade}

Em relação à área de ganho flexibilidade, McIntosh et al. (2001) comentam que, nessa área, há 5 oportunidades de melhoria: melhor resposta às necessidades do mercado; melhor acomodação das incertezas; melhor resposta a problemas de manufatura; melhor potencial para fornecer a mercados de baixo volume; e melhor potencial para ter maiores margens de lucro.

McIntosh et al. (2001) também comentam que a oportunidade de melhoria "melhor resposta às necessidades do mercado" é um dos mais importantes ganhos da redução do tempo de setup e é, frequentemente, a razão principal para se reduzir o tempo de setup. Nela, o ganho seria de se entregar os produtos no tempo que o cliente deseja e na quantidade que ele deseja sem a necessidade de se ter um grande inventário. No presente método, foi considerado que este ganho está sendo abordado na área "inventário reduzido", comentado no item 4.2. Tendo um alto inventário de todos os itens, também se poderia responder prontamente ao mercado, ou seja, a eliminação do acréscimo de inventário para se fornecer o mesmo tipo de serviço obtido pela redução do tempo de setup, a qual poderia ser considerada o ganho mensurável aqui procurado.

\subsection{Controle do processo}

Em relação à área de ganho "controle do processo", McIntosh et al. (2001) comentam que essa é uma área em que se tem dificuldade de avaliar os ganhos.

Eles comentam que é sabido que a redução do tempo de setup promove a redução de refugos e a melhoria da qualidade, mas há uma dificuldade de se quantificar para quanto irá o novo valor.

\subsection{Proposta de cálculo de ganho e abordagem para diminuição do tempo de setup}

Por meio dos comentários nos itens 4.1 a 4.5, estabeleceu-se que o presente método, apresentado na Figura 3, calculará o ganho:

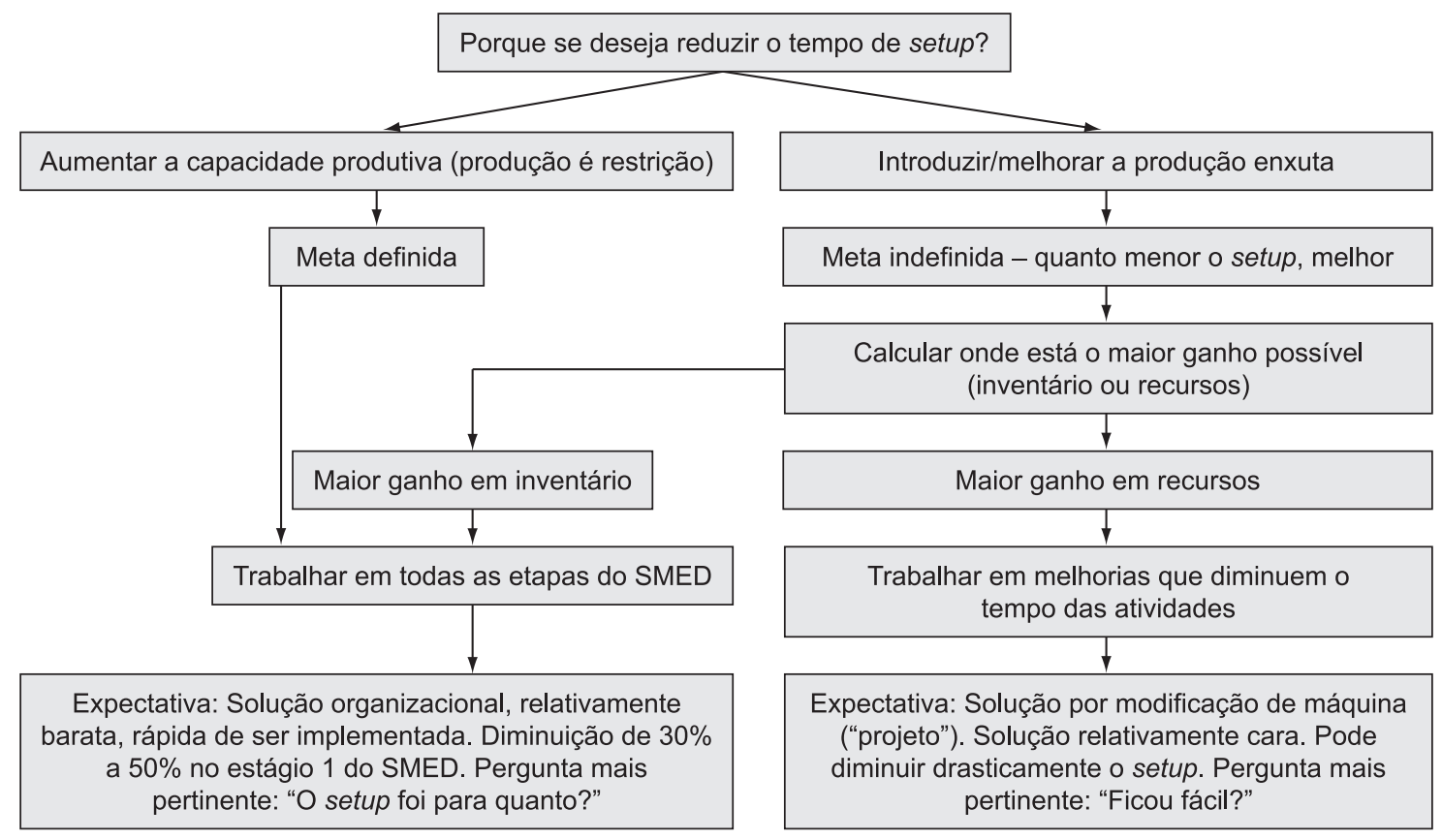

Figura 3. Método para o cálculo do ganho e definição da abordagem em trabalhos de redução do tempo de setup, ressaltandose o caminho quando mudança de máquina (projeto) é a primeira opção em um trabalho de redução de tempo de setup. 
- $\quad$ por aumento de capacidade, em função de uma demanda existente;

- pela redução de inventário; e

- pela redução de recursos.

A área "flexibilidade" foi incorporada à área "inventário", e a área "controle de processo" não está sendo considerada.

Além disso, a presente proposta sugere que, antes de se calcular o ganho nas três áreas acima, seja definida a motivação para a redução do tempo de setup, pois se observou que, além de se trabalhar na redução de tempo de setup para introdução/melhoria da produção enxuta, há uma outra motivação, que é aumentar a capacidade produtiva.

No caso da motivação para se reduzir o tempo de setup ser "problema de capacidade", o que é citado em trabalhos de Vollmann et al. (1988), Gilmore e Smith (1996), Clegg (1986), Klopsic e Houser (1997), o ganho pela redução do tempo de setup poderia ser a não compra de um novo equipamento.

No caso da motivação para se reduzir o tempo de setup ser "introdução/melhoria da produção enxuta", o que é citado em trabalhos de Sepheri (1987), Sheridan (1991), Johansen e Mcguire (1986), o ganho calculável poderia vir da diminuição dos estoques e redução de recursos.

Caso a motivação seja "aumentar a capacidade produtiva", ter-se-á uma meta e um ganho definidos (exemplo: a diminuição do tempo de setup em $30 \%$ evita o gasto de US\$ 500.000 na compra de uma nova máquina). Neste caso, tem-se a expectativa de que a solução possa vir de qualquer uma das fases do método SMED e, devido a Shingo (2000) pregar que no estágio 1 já se obtém uma redução no tempo de setup entre $30 \%$ e $50 \%$, há, portanto, grandes chances de que as soluções venham desse estágio e que seriam soluções de baixo custo por serem organizacionais e rápidas de serem implementadas.

Caso a motivação seja "introduzir/melhorar a produção enxuta", ter-se-á uma meta e um ganho inicialmente indefinidos, pois, neste caso, quanto menor o tempo de setup, melhor. Nessa situação, deve-se calcular o máximo ganho possível; para tanto, será considerado o impacto de um tempo de setup de zero minuto no estoque e na mão de obra. Essa fase tem como objetivo descobrir se vale a pena trabalhar em setup e qual seria a área de maior ganho. Deve-se ressaltar que o setup de zero minuto, utilizado nos cálculos, não é uma meta, é apenas uma maneira de se identificar o máximo ganho.

No caso da motivação ser "introduzir/melhorar a produção enxuta" e o maior ganho possível for a diminuição de inventário, deve-se trabalhar como quando se tem problema de baixa capacidade produtiva, ou seja, deve-se trabalhar para diminuir o tempo de setup, com a expectativa de que a solução possa vir de qualquer uma das fases do método SMED, e devido a Shingo (2000) pregar que na fase 1 já se obtém uma redução no tempo de setup entre $30 \%$ e $50 \%$. Há, portanto, grande chance de que as soluções venham desse estágio, que seriam soluções de baixo custo, por serem organizacionais, e rápidas de serem implementadas. Uma representação deste raciocínio pode ser vista na Figura 3.

No caso da motivação ser "introduzir/melhorar a produção enxuta" e o maior ganho possível for a redução de recursos, existe uma expectativa de que o trabalho venha a ser realizado no âmbito do estágio 3 do método SMED. Neste caso, não se trabalharia nos estágios 1 e 2 do método SMED, pois a separação do setup interno e externo e a transferência do setup interno para externo não diminuem a necessidade de recursos, afirmação esta confirmada pela Figura 2, que mostra que, nos estágios 0,1 e 2, a somatória dos tempos de setup internos e externos é constante, o que sugere que nesses estágios há, basicamente, uma transferência de atividades sem torná-los mais fáceis.

Esta diminuição do tempo para realização das atividades obtida no estágio 3 do método SMED se daria principalmente por uma mudança de equipamentos, diminuindo principalmente a atividade de ajuste, pois segundo Shingo (2000), esta atividade é a de maior duração dentro do setup.

Ao diminuir-se o tempo de setup por melhorias de equipamentos, haveria então a expectativa de que as soluções fossem relativamente demoradas para serem implementadas, e que fossem relativamente mais caras. Neste caso, uma pergunta normalmente feita para medir o sucesso do trabalho seria: "o setup ficou mais rápido?". Entretanto, essa pergunta precisa ser complementada por outra, ainda mais importante: "o setup ficou mais fácil?" Pois, o objetivo do trabalho, seria fazer ajustes com menos recursos.

\section{Estudo de caso}

Foi feito um estudo de caso em uma fábrica que trabalha em três turnos, 5 dias por semana, em uma unidade que tem 16 máquinas iguais, aqui denominadas máquinas $\mathrm{X}$.

As máquinas $\mathrm{X}$ tem um tempo de setup médio de 10,2 horas.

A fábrica tem, no total, 39 funcionários no chão de fábrica, 13 por turno, sendo que 8 são operadores e 5 são especialistas de setup.

Os operadores alimentam as máquinas com matériaprima, retiram os produtos prontos das máquinas e fazem medições para analisar a qualidade.

Os especialistas de setup fazem os setups e ajustes fora do setup (quando necessário).

O setup representa $13 \%$ do tempo total disponível para produção, não há problema de capacidade produtiva, inclusive são raros os momentos de horas extras em finais de semana e o giro de estoque é de aproximadamente 11,4 vezes ao ano. 
Os ajustes fora do setup representam $18 \%$ do tempo disponível.

A programação é um misto de fazer por pedido e previsão.

Há um plano para se implementar/melhorar a produção enxuta, mas para fazê-lo é necessária a redução do tempo de setup das máquinas $\mathrm{X}$, caso contrário pode haver problema de falta de capacidade produtiva e até mesmo, aumento de inventário.

Como primeira ação para reduzir o tempo de setup, foi utilizado o método SMED que se mostrou inadequado, como mostrado no item 5.1, e posteriormente se fez um cálculo de ganho pela entrada de uma nova tecnologia, que mostrou ser economicamente viável, e foi o caminho escolhido, como pode ser observado no item 5.2.

\subsection{Utilização do método Single Minute Exchage of Die}

Para a redução do tempo de setup foi escolhida uma das 16 máquinas $\mathrm{X}$ e nela foi aplicado o método SMED, cujos 4 estágios são descritos a seguir.

\subsubsection{Estágio 0}

Nesse estágio, como observado na Figura 1, o setup interno e o externo não estão identificados.

No trabalho realizado na máquina $\mathrm{X}$, foram feitos vários acompanhamentos para se compreender quais atividades fazem parte do setup, identificando-se o tempo gasto nessas atividades e classificando-as em setup interno e setup externo.

\subsubsection{Estágio 1}

Nesse estágio, como visto na Figura 1, deve-se retirar o setup externo do tempo de setup. O trabalho na máquina $\mathrm{X}$ mostrou que o setup interno representava $52,2 \%$ do tempo total de setup e o setup externo $47,8 \%$, mas, ao se tentar retirar o setup externo do tempo de setup, observou-se que $88,4 \%$ do setup externo era devido à falta de especialista de setup, ou seja, a máquina ficava parada por não se ter pessoas para fazer o setup, problema esse que tem como solução mais rápida a contratação de mão de obra. Entretanto, não fazia sentido adotar essa solução, pois não havia problema de falta de capacidade produtiva. Além disso, se fossem contratadas mais pessoas, o tempo de setup seria reduzido, podendo implicar em uma diminuição de inventário, caso fosse introduzida a produção puxada. Entretanto, cálculos mostraram que o gasto na contratação de mão de obra não seria pago pelo ganho resultante da diminuição do inventário. Logo, a solução não foi implementada e, por conseguinte, não foi possível reduzir o tempo de setup correspondente ao estágio 1.

\subsubsection{Estágios 2 e 3}

A atividade do estágio 2 é "transferir as atividades do setup interno para setup externo" e a atividade do estágio 3 é "racionalizar todos os aspectos do setup".

Como comentado por Shingo (2000), essas atividades podem ser feitas ao mesmo tempo.

No trabalho da máquina $X$, os estágios 2 e 3 foram realizados por um conjunto de pequenas ações, de custos relativamente altos que, ao final, diminuíram o tempo de setup de 10,2 horas para um pouco menos de 8 horas, o que foi considerado muito pouco pela própria expectativa gerada pelo método.

\subsubsection{Conclusão do trabalho de redução de tempo de setup na máquina $X$}

A grande propaganda de Shingo (2000) em relação ao SMED é a descoberta do setup interno e externo. A utilização dessa classificação não colaborou para aumentar a efetividade desse trabalho, pois não se tinha uma solução econômica para separar o setup interno e externo e havia poucas ações possíveis de conversão do setup interno para externo; logo, o método SMED não ajudou na diminuição do tempo de setup.

Surge então a pergunta: "Já que o método de redução de tempo de setup SMED não foi adequado, qual método deveria ser utilizado nos futuros trabalhos de diminuição do tempo de setup a fim de permitir a introdução e posterior melhoria da produção enxuta?"

\subsection{Foco em projeto}

Como alternativa às soluções organizacionais, havia a opção de se trabalhar em projetos. Por serem relativamente caros, pedem o cálculo do ganho para posteriormente se fazer uma análise de custo/ benefício. Para fins desta análise, seguiu-se o modelo apresentado na Figura 3.

Neste caso, a primeira questão a ser respondida é se a redução do tempo de setup tem como objetivo aumentar a capacidade ou implementar/melhorar a produção enxuta.

A motivação, no caso, é a introdução/melhoria da produção enxuta.

De acordo com a Figura 3, nesse momento deve-se calcular o maior ganho possível em inventário e em recursos.

A seguir serão mostrados os cálculos para os ganhos máximos.

\subsubsection{Cálculo do ganho máximo em inventário}

O cálculo do ganho máximo em inventário é realizado considerando-se que se terá um tempo de setup de zero minuto, e que não mais haverá 
programação por previsão, ou seja, a produção ocorrerá após a colocação de pedido ou para repor o estoque.

Será também considerado que o custo do inventário é dado pelo seu custo de capital.

Para se calcular o ganho máximo por redução de inventário, obteve-se a quantidade de produto no estoque e no processo e calculou-se qual seria a quantidade em um estado futuro, caso o tempo de setup fosse de zero minuto. Para realização destes cálculos, utilizou-se o método de cálculo de inventário em função do tempo de setup apresentado por Reis (2006), mas também comentado em Nishida (2006) e Smalley (2004).

Ao final, obteve-se uma redução de $75 \%$ do inventário, totalizando uma economia potencial de $\mathrm{R} \$ 79.200$.

\subsubsection{Cálculo do ganho máximo em recursos}

O cálculo do ganho máximo em recursos é realizado considerando-se um tempo de setup de zero minuto e que não há ajustes depois de finalizado o setup.

Essa última suposição (não haver ajustes após setup), considera:

- que a eliminação dos ajustes no setup também eliminará os ajustes fora do setup pelo simples fato de que os ajustes dentro do setup são mais difíceis, pois ocorrem em condições mais drásticas, ou seja, na mudança da fabricação de produtos diferentes; e

- que todos os ajustes que ocorrem fora do setup também ocorrem no setup, pois, se algo pode sair do padrão durante a produção, deve ser algo passível de um ajuste durante o setup.

O ganho máximo por meio de recursos é o custo dos funcionários que podem ser transferidos para outros setores da fábrica ao se atingir um tempo de setup de zero minuto.

Dessa forma, atingida a meta de redução de tempo de setup em aproximadamente $100 \%$, obter-se-iam os seguintes ganhos:

- A transferência de 15 especialistas de setup, pois não mais haveria setup e ajuste após setup. Vale salientar que o setup não é realizado pelos operadores e sim pelos especialistas, pois, quase na sua totalidade, a atividade de setup é de ajuste, atividade esta que exige um profissional com experiência; e

- A transferência de praticamente $1 / 3$ dos operadores (8 operadores) pela eliminação de um turno de produção de um total de três turnos, pois a soma da porcentagem de tempo de máquina parada por setup (13\%) e por ajustes após setup (18\%) é 31\% $(0,31=0,13+0,18)$, o que praticamente representa $1 / 3$ do tempo disponível para produção.

O teto de economia gerado pelas condições acima é de aproximadamente $\mathrm{R} \$ 1.138 .560,00 / \mathrm{ano}$, levando em consideração os custos dos especialistas e operadores.

\subsubsection{Teto de ganho e definição do escopo}

Para o cenário de tempo de setup zero minuto, obteve-se um teto de ganho para o inventário de $\mathrm{R} \$$ 79.200/ano e um teto de ganho de redução de recursos de $\mathrm{R} \$ 1.138 .560,00 /$ ano, totalizando-se um ganho de $\mathrm{R} \$ 1$ 1.217.760/ano.

Um gráfico dos possíveis ganhos pode ser visto na Figura 4.

Seguindo o método apresentado na Figura 3, como o ganho em recursos é consideravelmente maior que o ganho em inventário, tem-se a expectativa de que a solução venha por melhorias que diminuam o tempo das atividades dentro do setup, o que seria obtido por modificações de máquina ("projeto") que eliminem ou facilitem os ajustes, acabando com a necessidade da função "especialista de setup".

Decidiu-se então procurar por modificações de máquina que diminuíssem o tempo das atividades. Para estas modificações serem realizadas/ desenvolvidas, o gasto máximo por máquina teria um teto de aproximadamente $\mathrm{R} \$ 152.220$, pois, na empresa em estudo, considera-se que um projeto é economicamente viável se tiver retorno de investimento payback em 2 anos e $\mathrm{R} \$ 152.220=$ $2 \times \mathrm{R} \$ 1.217 .760 / 16$ máquinas.

Nesse caso, um objetivo inicial seria: encontrar soluções de engenharia que reduzam drasticamente o tempo de setup e que não peçam ajustes após o setup com um teto máximo de gasto de aproximadamente $\mathrm{R}$ \$ 150.000/máquina.

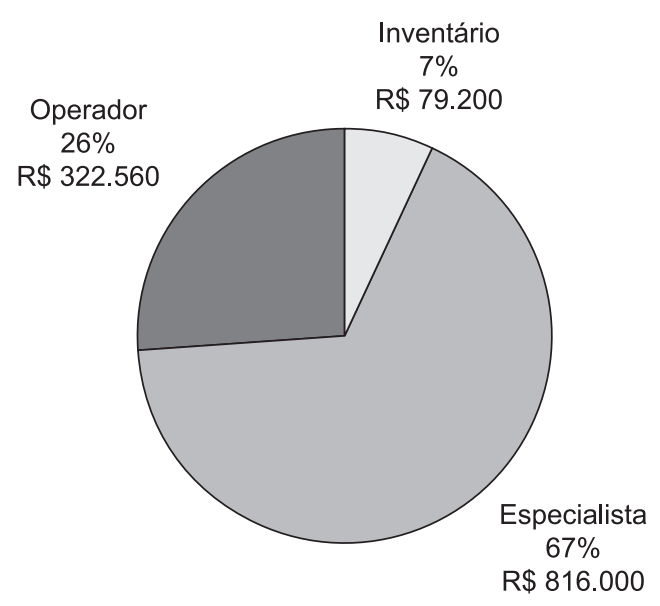

Figura 4. Economia anual em inventário e recursos pela redução do tempo de setup em $100 \%$. 


\subsubsection{Passos seguintes}

A máquina $X$ é relativamente longa, e nela ocorrem diferentes operações em 24 estações. Para que nela seja obtida uma redução de setup, foram desenvolvidas tecnologias que praticamente eliminam $72 \%$ dos ajustes dentro do setup e $58 \%$ dos ajustes fora do setup. Estas tecnologias estão em fase de implementação e têm um custo total/máquina de aproximadamente $\mathrm{R} \$ 35.000,00$, ou um custo total de implementação em 16 máquinas de $\mathrm{R} \$ 560.000,00$. As tecnologias basicamente reduzem as estações de trabalho para a metade, tornando as máquinas mais simples, o que possibilitará acabar com a função de especialista de setup, transferindo estes profissionais para outra função. Esta ação gera um ganho de aproximadamente $\mathrm{R} \$$ 816.000/ano, como comentado no item 5.2.3. Logo o payback do projeto será de menos de 1 ano.

Devido à introdução das novas tecnologias, além da eliminação da função de especialista de setup, estima-se obter uma redução do tempo de setup em aproximadamente $50 \%$.

\section{Discussão e conclusão}

No presente estudo de caso, tentou-se reduzir o tempo de setup utilizando-se o método SMED, que consiste basicamente na classificação das atividades em setup interno e externo, separação destas atividades e transferência das atividades de setup interno para setup externo. Este método propõe reduzir rapidamente o tempo de setup em até 50\% com ações de baixo custo.

$\mathrm{O}$ fato de não se ter obtido sucesso com o método SMED e se ter partido para um outro foco (mudança de projeto), gerou uma diminuição do moral do grupo, pelo simples fato de que na nova abordagem a expectativa é totalmente diferente: alto custo e longo tempo de implementação.

No estudo de caso, a mudança de foco para "projeto" se mostrou adequada e nele os projetos implementados têm uma relação custo/benefício adequada, sendo que, para o cálculo dos ganhos, foi utilizado o método aqui proposto, de cálculo de ganhos advindos de uma redução de tempo de setup.

O método proposto mostra como calcular os ganhos oriundos da redução do tempo de setup e também mostra a expectativa da abordagem inicial do trabalho (organizacional ou projeto), desta forma diminuindo a chance de se optar por um enfoque equivocado no início de um trabalho de redução de tempo de setup.

Além disto, o método proposto de cálculo de ganho por redução de tempo de setup prevê que nas fábricas com baixo custo de inventário e que tenham a função de especialista de setup, o enfoque inicial, mais adequado para a redução do tempo de setup, pode ser o desenvolvimento de novas tecnologias e não medidas organizacionais, que é o caminho mais comum e largamente utilizado.

De qualquer forma, a participação de operadores e especialistas de setup é fundamental para o atingimento de um bom resultado, o que só acontece em um ambiente de não demissão devido às melhorias implementadas. Para se realizar este intento, podem-se implementar algumas das 5 ações citadas no item 4.3 ou, simplesmente, pode-se optar por não repor funcionários que se aposentam ou tomam a iniciativa de trocar de emprego.

\section{Referências}

CLEGG, H. W. Operator machine studies technique reduces set-up time, implements JIT. Integrating Production \& Inventory Control, p. 52-53, 1986.

FABRY, R.; GELDERS, L. Cost/benefit analysis of a jit supply system in a car assembly plant. Logistic Information Management, v. 4, n. 4, p. 32-35, 1991.

GALLEGO, G.; MOON, I. Strategic investment to reduce setup times in the economic lot scheduling problem. Naval Research Logistics, v. 42, p. 773-790, 1995.

GILMORE, M.; SMITH, D. J. Set-up reduction on pharmaceutical manufacturing: an action research study. International Journal of Operations \& Product Management, v. 16, n. 3, p. 4-17, 1996.

HAY, E. J. Any machine setup time can be reduced by $75 \%$. Industrial Engineering, v. 19, n. 8, p. 62-67, 1987.

HUNTER, S. L. The 10 steps to lean production. FDM Management, p. 22-25, 2003.

JAPAN MANAGEMENT ASSOCIATION. Kanban: just in time at Toyota, management begins at the workplace. Tokio, 1988.

JOHANSEN, P.; McGUIRE, K. J. A lesson in SMED with Shigue Shingo. Industrial Engineering, p. 26-33, 1986.

KLOPSIC, A. R.; HOUSER, W. F. Increased throughput with rapid changeovers at tenneco. National Productivity Review, p. 59-65, 1997.

McIntosh, R. et al. An assesment of the role of design in the improvement of changeover performance. International Journal of Operations \& Production Management, v. 16, n. 9, p. 5-22, 1996.

McIntosh, R. I. et al. A critical evaluation of Shingo's SMEDS (Single Minute Exchange of Die). Int. J. Prod. Res., v. 38, n.11, p. 2377-2395, 2000.

McINTOSH, R. I. et al. Improving changeover performance: a strategy for becoming a lean, responsive manufacturer. Oxford: Butterworth Heinemann, 2001. $351 \mathrm{p}$.

MILEHAM, A. R. et al. Rapid changeover a pre-requisite for responsive manufacture. International Journal of Operations \& Production Management, v. 19, n. 8, p. 785-796, 1999.

MONDEN, Y. Toyota production system: an integrated approach to just-in-time. 3. ed. Norcross: Engineering \& Management Press, 1998. 480 p.

NISHIDA, L. D. Como determinar metas para o tempo de setup. Disponível em: <http://www.lean.org.br/bases. php?\&interno=artigo_18>. Acesso em: 04 maio 2006.

REIS, M. E. P. Proposta se um método racional para redução do tempo de setup: estudo de caso de uma empresa da indústria de produtos médico-hospitalares. 2006. 149 f. Dissertação (Mestrado)-Instituto Tecnológico de Aeronáutica, São José dos Campos, 2006. 
ROTHER, M.; SHOOK, J. Aprendendo a enxergar: mapeando o fluxo de valor para agregar valor e eliminar o desperdício. Versão 1.2. São Paulo: Lean Institute Brasil, 1999. 99 p.

SEPEHRI, M. P. E. Manufacturing revitalization at Harley Davidson Motor Co. Industrial Engineering, v. 19, n. 8, p. 26-33, 1987.

SHERIDAN, J. H. JIT spells good chemistry at Exxon. Industry week, p. 26, 1991.

SHINGO, S. Sistema de troca rápida de ferramenta: uma revolução nos sistemas produtivos. Porto Alegre: Bookman, 2000. 327 p.
SILVA, E. L.; MENEZES, E. M. Metodologia de pesquisa e elaboração de dissertação. 4. ed. Florianópolis: UFSC, 2005.

SMALLEY, A. Criando o sistema puxado nivelado: um guia para aperfeiçoamento de sistemas de produção, voltado para profissionais de planejamento, operações, controle e engenharia. São Paulo: Lean Institute Brasil, 2004. 114 p.

VOLLMANN, T. E. et al. Manufacturing planning and control systems. 4. ed. Irwin: Dow Jones, 1988. 904 p.

WOMACK, J. P.; JONES, D. T. A mentalidade enxuta nas empresas lean thinking. Rio de Janeiro: Campos, 2004. 408 p. 